\title{
LES DEUX METHODES DE DESCARTES
}

Descartes est célèbre pour avoir procuré, entre autres bienfaits intellectuels, une méthode nouvelle. II l'a proposée dans le fameux Discours de la méthode pour bien conduire sa raison et chercher la vérité dans les sciences (1637).

On prétend souvent qu'il y consigne sa méthode dans les quatre préceptes bien connus:

Le premier était de ne recevoir jamais aucune chose pour vraie, que je ne la connusse évidemment être telle: c'est-à-dire d'éviter soigneusement la précipitation et la prévention; et de ne comprendre rien de plus en mes jugements, que ce qui se présenterait si clairement et si distinctement à mon esprit, que je n'eusse aucune occasion de le mettre en doute.

Le second, de diviser chacune des difficultés que j'examinerais en autant de parcelles qu'il se pourrait, et qu'il serait requis pour les mieux résoudre.

Le troisième de conduire par ordre mes pensées, en commençant par les objets les plus simples et les plus aisés à connaître, pour monter peu à peu, comme par degrés, jusques à la connaissance des plus composés; et supposant même de l'ordre entre ceux qui ne se précèdent point naturellement les uns les autres

Et le dernier, de faire partout des dénombrements si entiers, et des revues si génerales, que je fusse assuré de ne rien omettre"1.

Ces "règles principales" de la méthode ont été universellement vantées, apprises, récitées - et l'on a même parfois tenté de les appliquer...

De grands penseurs ont désigné en elles la principale découverte philosophique de Descartes, et même la seule véritable, mais une découverte profondément révolutionnaire. L'un de ceux qui ont le plus exagéré, à cet égard, c'est Charles Péguy qui n'hésitait pas à écrire:

Dans ce discours de la méthode il n'y a qu'une partie sur six, la deuxième, qui soit des règles de la méthode. En tout, sept pages et demie. Et dans cette deuxième partie même il n'y a que le coeur, en

${ }^{1}$ Discours de la méthode - in Oeuvres complètes, col. Pléiade, Paris: Gallimard, 1937, pp. 103-104. 
tout vingt lignes, qui soit les règles de la méthode. Ce sont ces vingt lignes qui ont révolutionne le monde et la pensee $"$."

Eh bien, non! Vingt lignes n'ont pas "révolutionné le monde"! Ni même la pensée! Il faut pour cela plus que le discours ou l'oeuvre d'un homme. Mais Descartes a peut-être cédé lui-même quelque peu à cette illusion...

Du moins ne se contente-t-il pas de quatre préceptes! Le petit discours qu'il consacre à la méthode ne leur sert pas seulement d'accompagnement, ou d'assaisonnement. Sans quoi le Discours aurait été écrit pour rien, ou presque. Pour ce qui concerne véritablement la méthode, le lecteur n'a pas le droit de négliger ce qui prépare et précède l'exposé des quatre préceptes, ni ce qui le suit. Ces règles s'insèrent dans un développement dont elles ne sont qu'un moment, d'ailleurs particulièrement important. On ne peut les séparer sans danger de trahison, ni du Discours, ni de la première partie de celui-ci.

Dans cette premiere partie du Discours, on le sait, Descartes raconte sa vie. Faut-il prendre cela pour une simple coquetterie de grand savant ? De fait, si Descartes a cru éminemment utile, et même intellectuellement nécessaire de faire precéder ses grands ouvrages scientifiques d'une sorte de copieuse préface méthodologique et programmatique, il a aussi cru utile et nécessaire de commencer cette préface par une autobiographie. Il ne fait aucun doute que, pour lui, c'est le Discours tout entier qui revêtait une importance décisive, et aussi chacune des parties de ce Discours, y compris, bien sûr, la première!

Or il semble que tous les enseignements n'aient pas encore été tirés, de cette première partie; et, à la lumière de notre temps, certains d'entre eux peuvent se mettre mieux en valeur.

Loin de "révolutionner le monde", comme l'imaginait Péguy, les quelques préceptes de la méthode n'ont même pas réussi à régir la pensée de Descartes. De fait, ils ne contrôlent même pas le Discours où

2 Péguy, Note conjointe. Sur Bergson, p. 51. Dans sa Note sur M. Bergson et la philosophie bergsonienne, Oeuvres complètes. Paris: NRF, tome IX, 1924, p. 26. Péguy insiste: "Qu'est-ce qui fait la si haute et si grande et si juste fortune de la philosphie cartésienne? Ceux qui ont lu les oeuvres completes de Descartes ailleurs que dans les limpidités des manuels savent que toute la fortune de Descartes et de la philosophie cartésienne a été faite par quatre ou cinq lignes qui sont dans le Discours de la méthode. Et c'est tout. Et ces quatre ou cinq lignes, ces quatre ou cinq phrases sont précisément des préceptes pour ainsi dire de morale mentale, quelques principes antérieurs d'hygiène mentale, des règles de methode enfin, c'est lui qui le dit, non des principes ou des révélations ou des conclusions de systeme." 
ils apparaissent.

\title{
Descartes et sa "méthode"
}

La manière dont Descartes les introduit et en limite le nombre leur semble déjà tout-à-fait contraire. Il ne justifie cette parcimonie que par une vague inference analogique, juchée tant bien que mal sur un préjugé trivial, dans la plus coupable prévention et avec la plus grande précipitation:

\begin{abstract}
Et comme la multitude des lois fournit souvent des excuses aux vices, en sorte qu'un Etat est bien mieux réglé lorsque, nten ayant que fort peu, elles y sont fort étroitement observees, ainsi, au lieu de ce grand nombre de préceptes dont la logique est composé, je crus que j'aurais assez des quatre suivants..."3.
\end{abstract}

Rien de ce qui est affirmé là ne nous paraît évident, constatable, incontestable. On ne saurait céder plus complaisamment au préjugé politique et épistémologique de la simplicité! Si l'on voulait appliquer pointilleusement les préceptes de la méthode, si l'on voulait les "observer fort etroitement", alors l'analogie sur laquelle repose la limitation drastique de leur nombre pourrait même être dénoncée comme un cercle vicieux: ils s'établissent pratiquement sur une prévalence de la simplicité qu'ils ont pour tâche, entre autres, de justifier théoriquement.

S'il laisse ainsi jouer la désinvolture dans la fondation des préceptes, comment Descartes la contiendrait-il dans leur application ? Ne s'abandonne-t-il pas à la "précipitation" lorsque, faisant l'éloge des "fondements de sa physique", il prétend "qu'il ne faut que les entendre pour les croire", invoquant ainsi une évidence qui prive de tout intérêt la démonstration promise ensuite ?4 Peut-on donc encore croire quelque chose sans en avoir préalablement douté - si l'on obéit aux préceptes et si l'on s'est laissé pénétrer de l'esprit du Discours?

Descartes ne s'en tient d'ailleurs nullement aux préceptes dont il a délibérément limité le nombre. Chemin faisant, il en ajoute bien d'autres, et ces ajouts ne sont certes pas méprisables. Ainsi, il affirme l'exigence méthodique de rechercher toujours la causalité, et, à cet égard, de parler des hommes dans le même style que du reste, "c'est-à-dire en démontrant les effets par les causes, et faisant voir de quelles semences

\footnotetext{
${ }^{3}$ Discours de la méthode, op. cit., p. 103.

${ }^{4}$ Ibid., p. 138.
} 
et en quelle façon la nature les doit produire"ns.

La "production des effets à partir des semences", belle idée! Se trouvait-elle impliquée dans les préceptes ? Leur est-elle conforme ? S'accorde-t-il avec eux, ce point de vue génétique dont Descartes constate ailleurs la valeur et la fécondité: "La nature (des choses) est bien plus aisée à concevoir lorsqu'on les voit naître peu à peu en cette sorte que lorsqu'on ne les considere que toutes faites" ${ }^{16}$ ?

Pour ne pas compter parmi les "préceptes", voilà des indications quis'appliquent remarquablementà la procédure de Descartes lui-même, dans le Discours. il est bien plus aisé de concevoir la nature des règles "toutes faites", qui constituent la méthode proposée, dans la deuxième partie, lorsqu'on les a vu naître peu à peu, dans la première partie. On les comprend encore mieux lorsque Descartes nous dit, dans cette première partie aussi, de quelles semences ces préceptes ont été produits. Et Hegel, lisant tout cela, aurait dû jubiler. Le plus étonnant est qu'il soit resté impassible à cette lecture.

Il est assez facile de "relativiser" la portée des préceptes méthodiques, de montrer qu'ils ne satisfont pas au programme "absolutiste" que Descartes affiche ailleurs, et notamment dans son écrit antérieur, les Règles pour la direction de l'esprit. Pourquoi donc n'a-t-il pas achevé la rédaction de cet ouvrage, pourquoi n'a-t-il pas publié ce qu'il en avait composé ? Un des motifs de son abstention se trouve peut-être dans le constat de la distance qui les éloigne du Discours et de ses "préceptes". Ceux-ci reçoivent un complément et un éclairage très précieux de la publication posthume des Regles pour la direction de l'esprit, et l'on ne saurait estimer trop haut la chance, pour nous, de les avoir retrouvées. Mais, évidemment, leur confrontation avec le Discours marque le caractère relatif des deux écrits: deux étapes dans la progression d'une pensée sans cesse active, complexe, se mouvant à rythme syncopé.

Les préceptes du Discours dévoilent, mais en même temps dissimulent, un espace intellectuel beaucoup plus vaste que l'étroite plage sur laquelle ils concentrent leur faisceau lumineux. C'est un clair-obscur à la Rembrandt. Le génie de Descartes ne se manifeste pas tout entier en eux. Le Discours lui-même prend ses distances à leur égard. Ne pose-t-il pas comme condition pratique de leur usage, l'adoption d'une "morale par provision" élaboré toute entière sans eux, et même, on peut bien le dire, contre eux ? Dans l'imagination de cette morale par provision, la

\footnotetext{
${ }^{5}$ Ibid., p. 123.

I Ibid., p. 123.
} 
prévention, la précipitation et la confusion s'en donnent, si l'on ose dire, à coeur joie !

Il serait d'ailleurs intéressant d'examiner minutieusement leur rôle effectif et le degré de confiance qui leur est accordé dans les Traités scientifiques dont ils sont la preface et dont ils doivent recevoir une illustration exemplaire.

Mais, en tout cas, ne savons-nous pas qu'après tant d'années leurs conclusions sont périmées et que, même si leur contenu scientifique résultait d'une stricte observance des préceptes méthodologiques,ceux-ci n'en recevraient pour autant aucune confirmation décisive. Ils gardent pour nous l'intérêt historique d'une étape désormais franchie.

Si Péguy leur conférait une dignité exclusive, d'autres philosophes, et parmi les plus grands, les négligèrent entièrement. Dans leurs Leçons sur Descartes, Schelling et Hegel situent ailleurs le mérite du philosophe français: dans ce qu'ils tiennent pour son "système", et dans son idéalisme. Or les préceptes de la méthode ne conduisent pas par une sorte de nécessité au doute méthodique, encore moins au doute hyperbolique, au cogito, au dualisme et à toutes les conséquences que Descartes veut en tirer.

\section{La méthode constituante}

Quoi qu'il en soit de ces ajouts, de ces infidélités, de ces errements, Descartes a pourtant voulu proposer une nouvelle méthode, heuristique, prospective, normative. II a constitué cette méthode, il l'a codifiée et il l'a affichée dans le Discours.

Alors se pose à son égard une question véritablement fondamentale: comment Descartes a-t-il découvert ou inventé cette méthode, comment s'y est-il pris pour la constituer? Visait-il d'ailleurs comme une fin cette constitution, avant de l'accomplir ?

Descartes n'a évidemment pas découvert cette méthode en en suivant volontairement les préceptes, puisque ceux-ci n'étaient pas encore posés comme tels. Cela ne signifie pas pour autant qu'il ait procédé n'importe comment, d'une manière quelconque. Celui qui exhibe une méthode n'obéit pas toujours scrupuleusementà ses règles, mais celui qui croit procéder à l'aventure suit peut-être un chemin qui le mène quelque part, et l'on peut après coup apprécier l'intérêt du parcours.

La méthode active mais peu consciente grâce à laquelle on découvre une méthode que l'on pourra proclamer, se révèle peut-être plus intéressante que la méthode enfin constituée. Le comportement de Descartes, en cette affaire, révèle sa lucidité, sa tranquille audace 
intellectuelle, son génie.

D'abord, remarquons-le, il sait éviter le piège de la régression à l'infini. Si nous découvrons la méthode préalable qui permet de constituer la méthode achevée, il faudra découvrir la méthode antérieure qui a permis de découvrir cette méthode préalable, et ainsi, de méthode en méthode, avancer sans espoir de jamais aboutir...

Descartes saute par dessus cette difficulté, en effectuant ce qui pourrait paraître à beaucoup d'observateurs comme une sorte de coup de force, s'il ne le décrivait en termes si paisibles, et si vagues. II déclare en effet:

Je ne craindrai pas de dire que je pense avoir eu beaucoup d'heur de m'être rencontré dès ma jeunesse en certains chemins qui m'ont conduit à des considérations et des maximes dont j'ai formé une méthode..."?.

"Chemin, c'est méthode", comme le souligne Etienne Gilson dans son si précieux Commentaire du Discours de la methode ${ }^{8}$. Descartes "a été conduit", dans sa jeunesse, par "certains chemins", jusqu’à sa méthode constitué et proclamée. Ainsi distingue-t-il lui-même une méthode constituante - les chemins par lesquels il s'est laissé conduire et une méthode constitué, la méthode à laquelle ces chemins l'ont fait parvenir. L'art de Descartes consistera parfois, il faut bien l'avouer, à passer sans le dire d'une méthode à l'autre, ou même à les confondre.

Le mot méthode signifie originairement chemin et l'on remarque souvent l'usage fréquent par Descartes des mots qui, pour désigner le mouvement de la pensée, et ses conditions, évoquent le chemin, la route, la voie, le sentier, le carrefour, le cheminement, l'avancée, le parcours, la marche, la course, le pas, le détour, la ligne droite, l'itinéraire... Cette terminologie et ces images obsèdent véritablement Descartes. Notons des maintenant qu'elles se montrent tout aussi obsédante dans la Phénoménologie de l'Esprit de Hegel. Mais Hegel, bien sûr, marque mieux les caractères spécifiques d'un cheminement de la conscience, alors que Descartes, visiblement, s'empêtre dans les déterminations topographiques prises trop au sérieux.

Pour Hegel, qui dépasse l'image dès qu'elle est posée, et qui ne la pose que pour la dépasser, le chemin que la conscience parcourt dans

\footnotetext{
${ }^{7}$ Ibid., p. 92.

${ }^{8}$ Descartes, Discours de la méthode, édité et commenté par Etienne Gilson. Paris: Vrin, 1925, p. 258.
} 
sa formation est un chemin qui se construit et se parcourt lui-même, c'est-à-dire précisément tout autre chose que ce que l'on appelle ordinairement un chemin, et même son contraire.

Descartes appréhende lui-aussi, plus ou moins confusément, cette identité du chemin, du chemineau et du cheminement, dans le développement de sa propre conscience, mais, en quelque sorte, à côté de cette identité, il n'en maintient pas moins l'isolement dogmatique des déterminations mises ainsi en jeu.

\section{Ir conduite}

Dans le précepte cartésien: "Conduire par ordre mes pensées", - précepte essentiel à la méthode -, certains commentateurs, et parmi les plus prestigieux, retiennent surtout, et presque exclusivement, la notion d'ordre. Faut-il donc négliger les deux autres termes: "conduire mes pensées" ?

\section{Peut-on "conduire ses pensées" ?}

En relisant le Discours, on s'aperçoit que la pensée de Descartes, sur ce point, se conduit fort diversement et ne respecte aucun ordre des termes mis ainsi en relation. Ce flottement des déterminations ne laisse pas d'être instructif.

Il suggère certaines questions: Qu'est-ce que conduire ? Qui conduit? Qu'est-ce qui est conduit?

Parfois c'est la raison qui semble tenir les rênes: Descartes, par exemple, remarque que notre vie intellectuelle eût été beaucoup plus assurée et féconde "si nous avions eu l'usage entier de notre raison dès le point de notre naissance, et que nous n'eussions jamais été conduits que par elle"9. La raison peut-elle à la fois être au service d'un sujet, qui en fait usage, et "conduire" ce sujet, devenu en l'occurence son objet?

Dans le titre même du Discours, la raison accepte son rôle d'objet de la conduite: Discours de la méthode pour bien conduire sa raison..." Conjonction de mots très confuse, si l'on devait la prendre à la lettre: le conducteur, en cette affaire, ne serait pas la raison, et cependant c'est la raison qui tient la barre !

Diriger ses pensées, c'est vite dit. Qui dirige, sinon les pensées? Suis-je autre chose que mes pensées ?

Parfois Descartes ne livre les commandes ni à la raison, ni aux

9 Descartes, Discours de la méthode - in Oeuvres complètes, coll. Pléiade. Paris: Gallimard, 1937, pp. 99-100. 
pensées, ni au moi. C'est alors le chemin qui détient les prérogatives du sujet: "Certains chemins qui m'ont conduit..." Conducteur, vehicule, trajet échangent continuellement leurs rôles, pourtant distincts en principe. Mais le code de la route lui-même entre dans la danse. Qui donc commande ? Dans l'expression Discours de la méthode, le génitif est-il objectif ou subjectif ? Y parle-t-on de la méthode, ou bien est-ce la méthode qui prend la parole?

De fait, et bien que Descartes ne semble pas l'apercevoir nettement ni l'admettre expressément lui-même, c'est les deux. Il a le pressentiment de la proposition spéculative dans laquelle le sujet est l'objet, dans laquelle l'objet est la réflexion du sujet, ou inversement. La substance est sujet! Descartes ne l'affirme certes pas ainsi, mais il le sous-entend confusément lorsqu'il prend la résolution, selon ses propres termes, "de se conduire soi-même".

Il va désormais se prendre lui-même par la main.

Il avancera vers la connaissance parfaite avec une grande assurance, car il éprouve envers sa méthode une confiance entiere, on pourrait même dire : une confiance aveugle.

La méthode que Descartes se vante d'avoir trouvée et formée, est une méthode universelle, applicable, en tout temps, à tout et par tous. Elle mene infailliblement au but ceux qui se confient à elle.

Grâce à cette infaillibilité qu'elle se voit reconnue, la méthode benéficie d'une exceptionnelle promotion. Sans méthode, c'est l'errance, c'est le hasard et l'aventure. Avec la méthode, c'est la conduite ordonnée, l'ordre et la finalité. Elle est l'antihasard dans la recherche.

Cette conception de la méthode, Descartes la présente au début de la quatrième des Règles pour la direction de l'esprit. Il y condamne sans réserve l'enquête aventureuse qui ne devrait qu'à la chance ses rares succès. "Il est bien préférable, - dit-il -, de ne jamais chercher la vérité sur aucune chose plutôt que de le faire sans méthode ${ }^{n} 10$ !

"Or, ajoute-t-il, par méthode j'entends des règles certaines et faciles, grâce auxquelles tous ceux qui les observent exactement ne supposeront jamais vrai ce qui est faux, et parviendront sans se fatiguer en efforts inutiles mais en accroissant progressivement leur science, à la connaissance vraie de tout ce qu'ils peuvent atteindre ${ }^{n 11}$.

Quel confort! La méthode sait joindre l'agréable à l'utile.

De plus, elle se montre polyvalente, et absolue, origine ultime du

\footnotetext{
${ }^{10}$ Descartes, Oeuvres completes, op. cit., p. 14.

${ }^{11}$ Ibid.
} 
savoir:

Cette science doit en effet contenir les premiers rudiments de la raison humaine et n'avoir qu'à se développer pour faire sortir des vérités de quelque sujet que ce soit; et, pour parler librement, je suis convaincu qu'elle est préférable à toute autre connaissance que nous aient enseignée les hommes puisqu'elle en est la source ${ }^{12}$.

La méthode devient la Grande Cybernéticienne - ou, sur le mode de l'autre sexe, le Grand Timonier !

Certes, en passant des Règles dans le Discours, la méthode en rabattra quelque peu de ses prétentions premières, mais elle restera tout de même très orgueilleuse, très impérieuse, et, en tout cas, résolument normative: elle interdit les dérapages, elle balise le sens unique.

\section{La formation de la méthode}

On oppose ordinairement d'une manière radicale le constatif et le normatif: le fait n'est pas le droit, et l'on ne saurait confondre l'indicatif et l'imperatif, le concept et le mot d'ordre, le réel et le rationnel.

Pourtant ces distinctions, en elles-mêmes légitimes, ne valent que dans certaines limites, en certaines circonstances, abstraitement. De fait, l'esprit glisse sans cesse du constatif au normatif, et du normatif au constatif, et il ne s'en porte pas mal. La distinction dogmatique ayant été utilement posée, l'esprit trouve son avantage et son plaisir à la lever sans vergogne.

La Logique de Port-Royal l'admet paisiblement (1662): "Les hommes peuvent remarquer, en faisant des réflexions sur leurs pensées, quelle méthode ils ont suivie quand ils ont bien raisonné, quelle a été la cause de leurs erreurs quand ils se vent trompés, et former ainsi des règles sur ces réflexions pour éviter à l'avenir d'être surpris ${ }^{n 13}$.

Les hommes suivent donc d'abord une "méthode" sans le savoir, puis, après réflexion, ils forgent d'après elle des règles qu'ils suivront désormais volontairement. Nul autre que Bossuet ne sut le dire plus lapidairement: "... ils font une méthode réglée de leurs expériences". L'évêque de Meaux ne pouvait pas envisager la création d'une méthode ex nihilo par des créatures, s'agît-il même de Descartes!

\footnotetext{
12 Ibid., p. 16.

${ }^{13}$ Logique de Port-Royal, Premier discours, paragraphe 15.
} 
Mais Descartes avait bien volontiers admis, et le premier, que sa méthode normative et constituée dépendait d'une méthode constituante que l'on pouvait observer positivement: "Je pense avoir eu beaucoup d'heur de m'être rencontré dès ma jeunesse en certains chemins qui m'ont conduit à des considérations et des maximes dont j'ai formé une méthode..." ${ }^{14}$.

Gilson constate que les règles de la méthode cartésienne "ne font que codifier les démarches d'une raison exceptionnellement heureuse" et il décrit comme suit le processus de formation de ce qui sera la méthode constituée:

\begin{abstract}
Descartes interprète donc lui-même l'invention de la méthode de la manière suivante: 10. un heureux hasard ("fortunae auxilio..."; "je pense avoir eu beaucoup d'heur", consistant dans l'emploi spontané de certaines règles. 20 . la conscience de plus en plus claire de leur emploi jusqu'a l'elaboration des formules qui les définissent ("... unde paulatim animadverti... "; "... ut tandem animadverterim..." ; "... qui m'ont conduit à des considérations "); $3^{\circ}$ l'élévation de ces règles au rang de méthode ("... atque ita hanc totem methodum diligenter excolui...") ; ... et des maximes dont j'ai forme une méthode...". La traduction latine du Discours est encore plus précise: "... regularum sive axiomatum quibus constat Methodus..."
\end{abstract}

D'où l'interprétation de l'ensemble du texte : "Etant encore au collège, j'ai contracté certaines manières de réfléchir et de penser, qui devaient me conduire plus tard, en 1618-1619, aux considérations et aux maximes dont j'ai formé ma Méthode ${ }^{m 15}$.

Il y a donc des "manières de penser" qui "se contractent", comme des maladies; leurs séquelles produisent une méthode. Ne nous inquiétons pas du fait qu'une règle soit employée "spontanément", même si, d'ailleurs, nous opposons l'idée de régulation et de normativité à celle de spontanéité et de positivité !...

Effectivement, nous voyons s'effectuer une "élévation" (Aufhebung) du fait en norme, par une suppression de la forme du constat dans le maintien de son contenu, grâce à une prise de conscience des procédés réels de la pensée. Ni Descartes, ni Gilson, ne semblent apercevoir les implications contradictoires de cette opération, qui n'observe certes pas "fort étroitement" les règles de la méthode constituée. Mais Descartes, lui, accompli du moins l'opération impavidement,

\footnotetext{
${ }^{14}$ Descartes, op. cit., p. 92.

${ }^{15}$ Etienne Gilson, Commentaire du Discours, op. cit., p. 92.
} 
il suit hardiment une méthode constituante dont la dialectique est beaucoup plus vivante, complexe et subtile que la dialectique exténuée et sclérosée qui se maintiendra dans la méthode constituee.

Les règles ne pouvaient évidemment exister, en tant que telles, avant que ne fussent élaborées "les formules qui les définissent". Mais leur formulation, et leur fixation dans des formules, ne va pas sans quelque mutation, ni peut-être sans quelque sacrifice. Retraçant fidelement ce processus de formation de la méthode, Gilson en estompe les moments de rupture, de métamorphose qualitative, les sauts vertigineux: de l'inconscient au conscient, du spontané au volontaire, de l'historique à l'intemporel.

On aurait bien aimé que Descartes s'expliquât davantage sur les mutations intellectuelles qu'il provoque comme par magie; qu'il rendît mieux compte de la fluidité des notions et des concepts qu'il enferme finalement dans des distinctions figées; qu'il déployât plus lucidement la dialectique de la constitution de la méthode.

Concernant la production d'une méthode constituée, par la méthode constituante, la désignation peut varier: Descartes et Port-Royal disent former, Bossuet, faire; Gilson, codifier. Mais la signification de ces termes reste imprécise et ils n'apportent pas, en eux-mêmes, la signification théorique d'un glissement du positif au normatif pour des penseurs soucieux de distinguer absolument ces deux points de vue.

Dans le préambule du Discours, Descartes indique que la Seconde partie livrera "les principales règles de la méthode que l'auteur a cherchée" ${ }^{16}$. Il l'a cherchée! Mais pour cette recherche aussi vaut la remarque de Pascal: "Tu ne me chercherais pas si tu ne m'avais déja trouve". La quête de la méthode, le désir même de la trouver résultent de l'experience de toute une vie. L'auteur a 41 ans quand il publie le Discours. Le temps ne lui a pas manqué pour collecter les "considérations" et les laisser se métamorphoser, comme d'elles-mêmes, en "maximes".

Descartes signale toutefois la rupture entre le spontané et le réfléchi, le constatif et le normatif, et il la présente comme un acte de pure volonté: "Je pris un jour la résolution d'étudier aussi en moi-même, et d'employer toutes les forces de mon esprit à choisir les chemins que je devais suivre"17.

Jusqu'alors les chemins le menaient, mais à partir d'aujourd'hui,

${ }^{16}$ Descartes, op. cit., p. 91.

${ }^{17}$ Ibid., p. 98. 
c'est décidé, il choisira! Mais il n'a choisi, en fait, ni le jour, ni le lieu, ni le choix. Il n'a pas choisi de choisir. Il ne l'ignore pas: "Je me trouvais comme contraint, dit-il, d'entreprendre moi-même de me conduire" ${ }^{18}$.

\section{La chance de Descartes}

Pourquoi Descartes a-t-il suivi cette méthode spontanée particulière, cette méthode constituante singulièrement féconde qui devait le mener à sa célèbre méthode finalement constituée ?

Gilson répond sans hésitation: "C'est ainsi que Descartes (...) ayant rapporté d'avance l'honneur de ses succès sur sa méthode, ne s'attribue même pas le mérite de l'avoir inventée, mais en attribue la découverte à un heureux hasard ${ }^{19}$. Il ajoute:

En réalité, Descartes pense surtout à la chance qu'il avait eue de suivre dès son enfance la manière de raisonner la plus simple, la plus naturelle à l'homme, par là-même aussi, la plus féconde... La lumière naturelle retrouvait en lui la pureté qu'elle avait eue lors de la jeunesse du monde ${ }^{20}$.

A Descartes, tout arrive "par chance", comme en un rêve!

Gilson sent bien le paradoxe qu'il y aurait à trouver par aventure une méthode telle que celle à laquelle parvient Descartes. Mais il ne tente pas d'éclairer et de résoudre ce paradoxe, et il l'accentue encore, dans ce qu'il tient pour une boutade: "Descartes avait retrouve par hasard le secret de ne plus penser au hasard ${ }^{\mathrm{n} 21}$. Comment peut-il ne pas prendre plus au sérieux cette étrange situation, alors qu'il vient de rappeler qu"'il n'est pas dans les habitudes de Descartes d'attribuer au hasard les découvertes que fait l'esprit dans l'étude des sciences"22?

Gilson ne s'interroge pas sur le sens qu'il donne au mot hasard, en cette occurrence. Sans doute Descartes reconnaît-il lui-même, - et sur son aveu repose toute cette interpretation -, "qu'il pense avoir eu beaucoup d'heur de s'être rencontré, dès sa jeunesse en certains chemins..."23, mais les mots "heur" et "rencontre" renvoient ici plutôt à un avantage obtenu sans l'avoir expressément désiré et sans avoir

\footnotetext{
${ }^{18}$ Ibid., p. 102.

${ }^{19}$ Etienne Gilson, op. cit., p. 91.

${ }^{20}$ Ibid.

${ }^{21}$ Ibid.

${ }^{22}$ Ibid., p. 90.

${ }^{23}$ Descartes, op. cit., p. 92.
} 
mobilisé intelligemment les moyens adéquats pour l'obtenir, qu'à un hasard proprement dit qui consisterait, soit subjectivement en une ignorance des causes, soit objectivement en l'absence effective de telles causes, ou de conditions assignables.

De fait, la Première partie du Discours se propose presque exclusivement d'indiquer ces causes et ces conditions de la naissance de la méthode constituée, et ainsi expose-t-elle la méthode constituante. L"heur", la "chance" de Descartes, c'est que les situations, les caractères et les circonstances de sa vie l'ont mené précisément là où, rétrospectivement, il se félicite d'être parvenu. Ainsi la nécessité prend-elle parfois le visage du hasard, et, si ses effets se révèlent utiles et agréables, revêtelle la dignité d'une libre nécessité

Descartes est, avec Marx, l'un des rares philosophes qui ont tenu à rappeler, ne serait-ce que sommairement, l'histoire de leur pensée, à indiquer les conditions historiques générales et personnelles de l'apparition de leurs idées originales: comment l'esprit vient aux philosophes.

Certains interprètes de Descartes se sont émus du caractère exceptionnel de cette démarche. Alexandre Koyré s'exclamait: "Nous sommes trop accoutumés au Discours, accoutumés à y voir un grand philosophe nous raconter l'histoire de sa vie spirituelle. Cela nous paraît naturel et normal. Et nous ne voyons plus combien, au contraire, c'est insolite, inoui, surprenant ${ }^{\prime 24}$.

Mais d'autres, et parmi les plus prestigieux, sont enclins à réduire la portée ou même à contester l'intérêt de cette autobiographie cartésienne. Descartes ne deviendrait Descartes qu'en rompant avec sa vie ${ }^{25}$. L'ordre des raisons de la méthode constituée et du système qui en dérive, serait totalement indépendant de l'ordre historique des événements et des situations de la vie.

Dans ces conditions, la Première partie du Discours apparaît comme une simple manifestation de narcissisme, anecdotique et folklorique, à peine excusable chez un grand philosophe.

Or, visiblement, Descartes attribuait un grand intérêt à cette "histoire de sa vie", puisqu'il l'a écrite et qu'il l'a placée en tête de son Discours. Elle en constitue le projet fondamental et premier. L'auteur ne présente tout le Discours "que comme une histoire" ${ }^{\text {226, certes }}$

\footnotetext{
24 Cité par André Robinet dans son édition du Discours de la méthode. Paris: Nouveaux classiques Larousse, 1969, p. 126.

${ }^{25}$ Gueroult fait prévaloir une telle rupture en ce qui concerne Descartes. Althusser la détecte chez Marx.

${ }^{26}$ Descartes, op. cit., p. 93.
} 
exemplaire; il veut "y représenter sa vie comme en un tableau ${ }^{\text {n27. en }}$ tout cela il tient une promesse et réalise un programme, comme l'atteste la lettre que Guez de Balzac lui adressa en 1628:

Au reste, Monsieur, souvenez-vous, s'il vous plaît, De l'histoire de votre Esprit Elle est attendue de tous vos amis et vous l'avez promise (...). II y aura plaisir a lire vos diverses aventures dans la moyenne et dans la plus haute région de l'air, à considérer vos prouesses contre les Geants de l'Ecole, le chemin que vous avez tenu, le progrès que vous avez fait dans la vérité des choses"

Descartes décrit bien "le chemin qu'il a tenu", et c'est surtout pour se féliciter de la chance qu'il a eue d'être d'abord "tenu" par le chemin lui-même, - un bon chemin. Ce bonheur qui fut le sien, il l'analyse, il en détaille les profits et les agréments. Chemin faisant, - c'est le cas de le dire -, il s'arrête à toutes les circonstances favorables dans lesquelles le rôle de sa personnalité reste négligeable, mais qui, inversement, ont contribué à former cette personnalité. Il minimise, modestement, tout ce qui pourrait passer pour une contribution radicalement personnelle à ses découvertes majeures: "Je n'ai jamais présumé que mon esprit fût en rien plus parfait..."29.

Dans ces conditions, chaque élément constitutif de la "chance" globale de Descartes, et donc chaque évenement important de sa vie, apparaît à son tour comme une "chance". Ainsi Henri Lefebvre ne se retient-il pas d'écrire que "ce jeune gentilhomme poitevin, seigneur du Perron, appartenait à une famille de récente et de bourgeoise noblesse. Le hasard et la chance l'envoyerent au College des Jésuites de La Fleche où, dès 1813, si l'on en croit son biographe Baillet, il se prit à mépriser les livres, sans se croire pour cela autorisé à la paresse ou au mépris de la science" ${ }^{30}$.

Si cela est "le hasard et la chance", y a-t-il encore quelque chose qui leur échappe, dans la vie d'un homme et d'un peuple ? Mais si tout est hasard, il n'y a plus de hasard...

De fait, dans le Discours, Descartes passe en revue la plupart des conditions assignables qui devaient être réunies pour qu'il devint philosophe, l'inventeur d'une méthode nouvelle. Il veut voir clair en son coeur.

Mais en même temps, comme le souligne M. Henri Gouhier

\footnotetext{
${ }^{27}$ Ibid.

${ }^{28}$ Lettre du 30 mars 1628 , cité par E. Gilson, op. cit., p. 98.

29 Descartes, op. cit., p. 92.

${ }^{30}$ Henri Lefebre, Descartes, Ed. Hier et Aujourd'hui. Paris: 1947, p. 9.
} 
"L'histoire de l'esprit de Descartes devient exemplaire, et, comme telle, quitte la biographie de $\mathrm{M}$. Descartes pour tracer l'itinéraire recommandé à quiconque entend suivre la raison"31.

De toute évidence, la plupart des situations et des circonstances que Descartes énumère et décrit, concernent en effet une pluralité d'individus, tout un groupe social. Bien d'autres que lui étaient nés en France, au tournant du siecle, dans des provinces semblables à la Touraine. Les "familles de récente et bourgeoise noblesse" mettaient allegrement de nouveaux enfants au monde et, l'âge venu, c'est elles qui les envoyaient - non "le hasard et la chance" - au College des Jésuites de La Fleche ou en d'autres bonnes maisons d'éducation et d'instruction. Gilson le fait remarquer: le "curriculum vitae" de Descartes n'a rien d'extraordinaire ${ }^{32}$, il caractérise le mode de vie et de formation des individus relevant d'un milieu social définissable.

Descartes suggère aussi, et à juste titre, l'existence et l'efficacité de son apport personnel. Des circonstances particulieres lui ont permis de prendre conscience mieux que d'autres d'une situation générale et d'en exploiter plus aisement, au profit de la science, les enseignements et les avantages. Sa fausse modestie ne l'empêche pas tout-à-fait d'accorder de l'importance à son idiosyncrasie intellectuelle. Au point même de prétendre, là encore d'une manière paradoxale et sans doute quelque peu ironique, qu'en fin de compte, bien qu'il n'ait "jamais fait beaucoup d'état des choses qui venaient de son esprit"133 ... "s'il y a au monde quelque ouvrage qui ne puisse être si bien achevé par aucun autre que par le même qui l'a commence; c'est celui auquel je travaille"134. Il ne veut "conseiller à personne de l'imiter" ${ }^{35}$ !

Mais il n'en oublie pas pour autant le fonds commun dans lequel il a puisé, la situation générale qui le porte, avec tant d'autres qui vivent, pensent et travaillent en même temps que lui.

Si un nombre significatif d'amis, de témoins, de lecteurs ont pu le comprendre et le suivre dans des démarches inouies, révolutionnaires, et même sacrilèges au regard de quelques uns, c'est bien qu'ils étaient eux-aussi orientés dans le même sens par l'expérience de leur vie, et, pour employer une expression commode, soulevés par la même épistémie nouvelle, emportés par le même esprit du temps.

\footnotetext{
${ }^{31}$ Cité par André Robinet, op. cit., p. 126.

${ }^{32}$ Etienne Gilson, Commentaire du Discours, op. cit., p. 144.

${ }^{33}$ Descartes, op. cit., p. 134.

${ }^{34}$ Ibid., p. 141.

${ }^{35}$ Ibid., p. 101.
} 
En contrepartie, nombreux restaient les démunis, ceux que la même "chance" ne favorisait pas, ceux que leur situation sociale tenait à l'écart de la culture et de ses possibilités de renouvellement: les Grands, les paysans, les manouvriers...

La "chance" de Descartes, ce fut la chance d'être Descartes en son temps et sur son continent. Ce serait faire un singulier abus du mot hasard que de l'appliquer, sans autre justification ou explication, à la vie entière d'un homme, d'une nation, d'une époque. En ce qui concerne Descartes, on peut aussi bien parler de sa "libre nécessité" ou de son destin.

Dans la Première partie du Discours de la Méthode, et aussi, çà et là, dans le Discours tout entier, l'auteur analyse longuement les composantes nécessaires du processus de sa formation. Chacun est ce qu'il est devenu. Et la méthode aussi a dû devenir ce qu'elle est, par les voies d'une autre méthode. La révolution de la pensée, proclamée, a été suscitée par une révolution silencieuse, dans le monde. Comme le dit Hegel: "L'interêt de la biographie a l'air de s'opposer directement à un dessein général; mais elle a elle-même comme arrière-plan le monde historique auquel l'individu est mêlé".

\section{La dialectique de Descartes}

Hegel définit sa Phénoménologie de l'Esprit comme "la science de l'expérience de la conscience", et il précise ainsi le but poursuivi: "La tâche de conduire l'individu de son point de vue inculte jusqu'au savoir, devait être entendue en son sens universel et l'individu universel, l'esprit conscient de soi, devait être considéré dans son processus de culture" ${ }^{136}$.

On peut dire que le Discours de la Méthode accomplit, très sommairement, cette tâche, mais à sa manière: seulement en ce qui concerne l'individu singulier. Il rend compte de l'expérience particulière et du chemin de culture de la conscience de Descartes: depuis l'enfance inculte jusqu'au savoir absolu, jusqu'au savoir scientifique et au savoir de Dieu, en passant par les étapes de l'illusion et de l'erreur, du doute et du désespoir, de la contradiction, de l'abstraction, de l'ironie... Descartes règle ses comptes avec toutes les formes préalables de la conscience et de la connaissance philosophiques comme le fera plus tard Hegel: en les décrivant, en décrivant leurs dépassements successifs, en les présentant

\footnotetext{
${ }^{36}$ Preface de la Phenoménologie, Edition bilingue par J. Hyppolite. Paris: Aubier, 1966, p. 69.
} 
comme les moments d'un processus global.

Il est regrettable que la description reste aussi succincte et individuelle, mais on ne peut négliger, à cause de cela, ni sa surprenante présence, ni sa ressemblance avec la description hégélienne.

Elle offre d'ailleurs un caractere ambigu. Pourquoi Descartes l'aurait-il publiée, si elle ne présentait un intérêt plus général ? Le Discours décrit, mais, comme la Phénoménologie, il enseigne en décrivant. Descartes le qualifie de "fable", parce que la fable a un caractere didactique ${ }^{37}$ : fabula docet.

Dans la Phénoménologie, le chemin de l'expérience (Erfahrung) qui est décrit, reste plus individuel et personnel que Hegel ne le souhaite. Dans le Discours, il a une valeur plus générale que Descartes ne le croit: l'auteur n'y retient que ce qu'il juge essentiel au mouvement de pensée aboutissant à la constitution de la méthode.

La Phénoménologie de Hegel, envisagé par rapport à l'histoire réelle de l'esprit humain et du monde, est, elle-aussi, une sorte de "fable" - et sans doute encore plus fabuleuse - en un autre sens du mot -, que le récit cartésien. Qu'il s'agisse, en tout cas, dans le Discours, d'un combat pour se faire reconnaître et pour gagner les "batailles" de la vérité, l'auteur en a pleinement conscience, et ne le dissimule pas, et il déploie devant nos yeux les figures successives de son combat.

La méthode de Descartes est dialectique, puisque tout est dialectique. Encore convient-il de déceler le genre de dialectique dont elle releve.

La dialectique s'exerce à divers niveaux et sous diverses formes, et, chez Descartes, elle obtient une grande efficacité. La fécondité, en même temps que l'audace et la subtilité de la dialectique cartésienne se marquent déjà, par exemple, dans le jeu de ses deux méthodes, dans leur distinction et dans la succession de leurs interventions, mais aussi dans ce saut intellectuel du normatif au positif, et vice-versa, qui permet à Descartes de réunir et même d'unifier profondément les deux méthodes. Mais il serait aisé de mettre en évidence une telle dialectique de haut niveau en bien d'autres endroits du Discours. Les deux méthodes de Descartes ne nouent pas seulement entre elles des relations dialectiques, elles sont aussi dialectiques chacune pour soi.

La méthode constituée recueille des préceptes qu'aucun dialecticien ne saurait négliger, car leur respect commande tout exercice conscient de la dialectique et en définit quelques opérations indis-

${ }^{37}$ Descartes, op. cit., - Voir Gilson, op. cit., p. 99. 
pensables: distinguer, séparer, déterminer clairement et distinctement, réfléchir, éviter les préjugés, ne pas se laisser séduire par les apparences, chercher et établir un ordre, énumérer, récapituler... Où donc Hegel a-t-il jamais désavoué de pareilles règles ? Quel penseur sérieux pourrait se dispenser de les respecter ?

Toutefois, elles ne constituent, dans leur nécessité, qu'un moment, certes décisif, de la dialectique consciente - tout en contenant d'ailleurs les autres moments en puissance. Elles restent unilatérales, partielles et partiales. Le dialecticien se doit de les restituer à la fluidité de la pensée active et de la vie. Ce processus fluide, Descartes l'a décrit, et l'a fragmentairement analysé dans la présentation de sa méthode constituante, dans l'exposé de la genèse de ses idées, accompagnant l'histoire de sa vie.

La méthode constituante manifeste une dialectique en acte, qui se fige ensuite et s'isole abstraitement dans la méthode constituée. Dans sa positivité (au sens hégélien du terme), la méthode constituée perd en richesse dialectique, mais elle gagne une conscience et une intentionalité plus hautes. Chez Descartes, plus la dialectique est active et féconde, moins elle est consciente de soi. Elle joue au plus haut degré dans l'ironie cartésienne, mais c'est une ironie dont l'auteur lui-même semble parfois être dupe.

Il en va de Descartes comme de la plupart des prédécesseurs de Hegel: la vigueur de leur dialecticité varie en raison inverse de la conscience qu'ils en prennent. Presque tous ceux dont Hegel revendique l'héritage ont été dialecticiens sans le savoir.

On doit donc, en pensant la dialectique avec les moyens de l'entendement, - comme il est impossible de ne pas le faire, et comme Hegel lui-même en a donné l'exemple -, distinguer artificiellement, du point de vue de la conscience, trois types de dialectique. D'abord, une dialectique réelle, en laquelle s'expriment les lois les plus générales du devenir de tous les êtres, et qui n'est pas nécessairement consciente en aucun d'eux. Puis une dialectique consciente, c'est-à-dire la dialectique d'une conscience, dans son travail de production comme dans son travail de représentation - mais cette conscience ne se rend pas nécessairement compte de ce qu'elle fait, ni de ce qu'elle est. Enfin une dialectique consciente de soi, sachant ce qu'elle fait, capable de dire ce qu'elle est. Au plus haut niveau de conscience de soi et de lucidité théorique, on la trouve chez le grand Hegel.

Chez Descartes, elle n'atteint que le niveau de la conscience spontanée, et elle s'y exerce admirablement, mais elle ne le sait pas. La pensée de Descartes se meut avec habileté, souplesse et élégance dans 
les contradictions les plus fécondes, mais sans les reconnaître et les avouer comme telles.

Il n'est donc pas question de dresser une opposition dogmatique entre méthode cartésienne et méthode dialectique hégélienne. Il ne manque certes pas de differences entre les deux! Mais ce sont des différences dialectiques qui, transposées dans le temps, se traduisent en étapes d'un développement. Les méthodes cartésiennes jalonnent un chemin qui conduit au delà-d'elles. 